M. Castaing $\cdot$ A. Guerci $\cdot$ J. Mallet $\cdot$ P. Czernichow $\cdot$

P. Ravassard $\cdot$ R. Scharfmann

\title{
Efficient restricted gene expression in beta cells by lentivirus-mediated gene transfer into pancreatic stem/progenitor cells
}

Received: 20 September 2004 / Accepted: 4 December 2004 / Published online: 10 March 2005

C) Springer-Verlag 2005

\begin{abstract}
Aims/hypothesis: Gene transfer into pancreatic beta cells, which produce and secrete insulin, is a promising strategy to protect such cells against autoimmune destruction and also to generate beta cells in mass, thereby providing a novel therapeutic approach to treat diabetic patients. Until recently, exogenous DNA has been directly transferred into mature beta cells with various levels of success. We investigated whether exogenous DNA could be stably transferred into pancreatic stem/progenitor cells, which would subsequently differentiate into mature beta cells expressing the transgene. Methods: We designed transplantation and tissue culture procedures to obtain ex vivo models of pancreatic development. We next constructed recombinant lentiviruses expressing enhanced green fluorescent protein (eGFP) under the control of either the rat insulin promoter or a ubiquitous promoter, and performed viral infection of rat embryonic pancreatic tissue. Results: Embryonic pancreas infected with recombinant lentiviruses resulted in endocrine cell differentiation and restricted cell type expression of the transgene according to the specificity of the promoter used in the viral construct. We next demonstrated that the efficiency of infection could be further improved upon infection of embryonic pancreatic epithelia, followed by their in vitro culture, using conditions that favour endocrine cell differentiation. Under these conditions, endocrine stem/progenitor cells expressing neurogenin 3 are efficiently transduced by recombinant lentiviral vectors.
\end{abstract}

M. Castaing $\cdot$ R. Scharfmann $(\bowtie)$

INSERM EMI 0363, Faculté Necker,

156 rue Vaugirard,

75015 Paris, France

e-mail: scharfmann@necker.fr

Tel.: +33-1-40615565

Fax: +33-1-43060443

A. Guerci $\cdot$ J. Mallet $\cdot$ P. Ravassard

CNRS UMR 7091, Hôpital Pitié Salpêtrière,

Paris, France

P. Czernichow

INSERM U457, Hôpital Robert Debré,

Paris, France
Moreover, when eGFP was placed under the control of the insulin promoter, $70.4 \%$ of the developed beta cells were eGFP-expressing cells. All of the eGFP-positive cells were insulin-producing cells. Conclusions/interpretation: We have demonstrated that mature rat pancreatic beta cells can be stably modified by infecting pancreatic stem/progenitor cells that undergo endocrine differentiation.

Keywords Beta cells - Gene transfer - ngn3 Recombinant lentivirus $\cdot$ Stem/progenitor cells

Abbreviations CMV: cytomegalovirus $\cdot \mathrm{CPA}$ : carboxypeptidase A - E: embryonic day - eGFP: enhanced green fluorescent protein - HBSS: HEPES buffered saline solution - ISH: in situ hybridisation - ngn3: neurogenin 3 . RIP: rat insulin II gene promoter - scid: severe combined immunodeficiency - TU: transduction unit - VSV: vesicular stomatitis virus

\section{Introduction}

Type 1 diabetes mellitus is due to an autoimmune process resulting in the destruction of pancreatic beta cells, the cells that produce and secrete insulin. Replacement of insulinproducing tissue by transplantation of mature beta cells represents an effective method of curing type 1 diabetes. However, many obstacles remain before cell replacement therapy [1] can be used routinely to treat diabetic patients [2]. The major issues to be solved are allorejection, recurrence of anti-islet cell autoimmunity and the shortage of available donor tissue.

To address the immunological problems and increase the limited supply of human tissue available for grafts, attempts have been made to transfer exogenous DNA into mature beta cells. Several gene delivery viral systems, such as moloney-based retroviral vectors, adenoviral vectors, adenoassociated viral vectors, or lentiviral vectors have been employed for ex vivo gene transfer into pancreatic islet cells. Moloney-based retroviral vectors were inefficient in transducing mature insulin-expressing cells due to the lack 
of proliferation of mature beta cells [3]. Adenoviruses were highly efficient in transducing non-dividing beta cells [35], however, the expression of the transgene was transient. This could be due either to the absence of genome integration of the virus or to the elimination of transduced cells by the host immune system [6]. To circumvent this limitation in long-term expression, adeno-associated viral vectors have been used to transduce pancreatic islet cells $[7,8]$. Lentiviral vectors were capable of transducing mature islet cells with sustained expression of either reporter genes [3, 9, 10], therapeutic molecules [10], or immortalising genes [11]. Together, the above data indicate that exogenous DNA can be stably transferred to mature beta cells $[3,9,10]$, providing limited protection against immune destruction [10]. However, this approach failed to generate beta cell lines [11].

In the aforementioned studies, only mature beta cells were infected and transduced with various viral vectors. In cell types of the haematopoietic system, mature cells have been modified by viral infection of progenitor cells that undergo differentiation. Several groups have recently reported successful transduction of reporter genes or genes of therapeutical importance into progenitor cells that were taken from umbilical cord blood or bone marrow and differentiated into mature cells [12-15]. In these experiments, the transgene was constitutively expressed, irrespective of the differentiation stage. More recently, Pawliuk and co-workers further modified the system using celltype-specific promoters [16]. They infected haematopoietic stem cells with recombinant lentiviruses expressing their gene of interest under the control of the beta globin locus control region, achieving high erythroid-specific gene expression levels [16].

While the results described above concern the haematopoietic system, only a limited number of studies in solid tissue show stable modification of mature cells by transduction of progenitor cells [17-19]. However, in these reports, only constitutive promoters were used, resulting in an expression of the transgene without cell type restriction.

Our objective was to determine whether beta cells can be modified by infecting stem/progenitor cells that will differentiate into beta cells. For this purpose, we designed ex vivo experimental models in which immature embryonic pancreases, rich in endocrine progenitors, develop into mature pancreatic tissue. We next analysed conditions to infect immature pancreatic tissues with recombinant lentiviruses expressing enhanced green fluorescent protein (eGFP) under the control of the insulin promoter. Our data demonstrate that recombinant lentiviruses can infect pancreatic stem/progenitors, such as cells positive for neurogenin 3 (ngn3) that will differentiate into beta cells expressing the transgene of interest. This strategy represents a new approach to the generation of genetically modified beta cells.

\section{Materials and methods}

DNA constructs and recombinant lentiviral production The backbone of the lentiviral construct, pTRIP, has been previously described [20]. The vector, pTRIP $\Delta U$ U.CMVeGFP [17], expresses the $e G F P$ gene under the control of an internal cytomegalovirus (CMV) promoter. A new lentiviral vector, pTRIP $\Delta$ U3.RIP405-eGFP, was constructed with the Rat insulin II gene promoter (RIP) in order to restrict the expression of $e G F P$ to insulin-producing cells. A 405-bp fragment of RIP was amplified from the PBSRIP-beta globin plasmid (kindly provided by P. Herrera, University of Geneva, Switzerland) [21] using the PCR expand system (Roche Diagnostic, Meylan, France) with the following primers: $M l u I$ RIP405 sense 5'cgacgcgtGGAC ACAGCTATCAGTGGGA3' and BamHI RIP antisense 5'cgggatccTAGGGCTGGGGGTTACT3'.

The resulting PCR product was subcloned into the pGEMT-easy vector (Promega, Charbonnières, France) and the inserted RIP405 fragment was entirely sequenced. The $M l u \mathrm{I}-$ Bam HI digestion fragment was then inserted into a $M l u \mathrm{I}-$ Bam HI linearised promoterless pTRIP $\Delta \mathrm{U} 3$.-eGFP vector (kindly provided by Dr A. P. Bemelmans, CNRS UMR 7091, Paris).

Lentiviral vector stocks were produced by transient transfection of 293T cells with the p8.7 encapsidation plasmid $(\Delta \operatorname{Vpr} \Delta \operatorname{Vif} \Delta \mathrm{Vpu} \Delta \mathrm{Nef})$ [22], pHCMV-G, encoding the vesicular stomatitis virus (VSV) glycoprotein-G [23] and the pTRIP $\Delta \mathrm{U} 3$. recombinant vector as previously described [20]. The supernatants were treated with DNAse I (Roche Diagnostic) prior to ultracentrifugation and the resulting pellet was resuspended in PBS, aliquotted and frozen at $-80^{\circ} \mathrm{C}$ until use. The amount of $\mathrm{p} 24$ capsid protein was quantified by the HIV-1 p24 ELISA antigen assay (Beckman Coulter, Villepinte, France).

Promoter specificity in a lentiviral context: titrations and transduction efficiency The titration of each vector stock was determined by infecting Min6 and 293T cells. Min6 cells, derived from a mouse insulinoma [24], and 293T cells, derived from human kidney, were grown at $37^{\circ} \mathrm{C}$ in DMEM (Invitrogen Life Technologies, Cergy-Pontoise, France) supplemented with $15 \%$ and $10 \%$ heat-inactivated fetal calf serum, respectively. Cells were plated in 24-well plates and kept in contact with increasing amounts of each lentiviral suspension for $1 \mathrm{~h}$ with diethylaminoethyl-dextran $10 \mu \mathrm{g} / \mathrm{ml}$ (Sigma-Aldrich, Saint-Quentin Fallavier, France) [25]. The virus-containing medium was then removed and fresh medium was added. After a 72-h incubation period, cells were washed with PBS, detached with trypsin and fixed in $1 \%$ formaldehyde-PBS. The number of GFP-positive cells was analysed using a flow cytometer (FACScan; Becton Dickinson, Le-Pont-de-Claix, France). Acquisition data were processed with CellQuest for Macintosh. Transduction units (TU) were calculated using the formula:

$\mathrm{TU}=f_{\mathrm{eGFP}} C_{0} V$

where $f_{\mathrm{eGFP}}$ is the frequency of eGFP-positive cells determined by FACScan, $C_{0}$ is the total number of target cells infected and $V$ is the volume of the viral stock used. 
The eGFP titres were analysed for several virus dilutions. Each point was performed in triplicate. The mean titre values were calculated for the viral dilutions corresponding to $1-20 \%$ of transduced cells in order to avoid multiple infection events.

Preparation of pancreatic rudiments Pregnant Wistar rats were obtained from Janvier (CERJ, Le Genest, France). All animal manipulations were performed according to the guidelines of the French Animal Care Committee. The morning post coitum was designated as embryonic day (E) 0.5. At E13.5 and E16.5 days of gestation, pregnant female rats were killed by $\mathrm{CO}_{2}$ asphyxiation. Rat embryos were removed from the uterus, the digestive tracts isolated and the pancreatic primordia dissected [26]. Intact or partially dissociated embryonic pancreases were prepared for transplantation. The pancreatic buds were incubated with $0.16 \mathrm{mg} / \mathrm{ml}$ of Dispase I (Roche Diagnostic) for $20 \mathrm{~min}$ and subsequently mechanically dissociated. The purified pancreatic epithelium used for organotypical culture was separated from its surrounding mesenchyme by enzymatic and mechanical treatments as previously described $[27,28]$.

Infection of embryonic pancreases and transplantation Recombinant lentiviruses were used to infect either intact or partially dissociated pancreases. Tissues were incubated with viral particles (120 ng of p24) for $2 \mathrm{~h}$ at $37^{\circ} \mathrm{C}$ with $5 \% \mathrm{CO}_{2}$ atmosphere, in $200 \mu \mathrm{l}$ of RPMI-1640 medium (Invitrogen) supplemented with 10\% heat-inactivated fetal calf serum containing HEPES (10 mmol/l), L-glutamine ( $2 \mathrm{mmol} / \mathrm{l}$ ), non-essential amino acid (Invitrogen), penicillin $(100$ units $/ \mathrm{ml})$ and streptomycin $(100 \mu \mathrm{g} / \mathrm{ml})$. To increase the viral infection efficiency, diethylaminoethyldextran was added to a final concentration of $10 \mu \mathrm{g} / \mathrm{ml}$. After lentiviral infection, tissues were washed twice with HEPES buffered saline solution (HBSS, Invitrogen) and kept on ice until transplantation into severe combined immunodeficient (scid) mice.

Seven-week-old, male scid mice (Charles River Laboratories, L'arbresle, France) were maintained in isolators.

Using a dissecting microscope, the pancreases were implanted under the kidney capsule as previously described [29], with the following modifications. The left kidney was exteriorised; a small transverse incision was made through the capsule on the ventral surface of the kidney, near the inferior pole. A small silicon cylinder was pushed under the capsule to provide a sealed space to confine the transplanted cells and tissues [30]. The tissues were then introduced into the cylinder using forceps and/or a Hamilton syringe. At different time points after transplantation, the mice were killed, the kidney removed, and the graft dissected. Fixation with $3.7 \%$ formaldehyde was used prior to embedding in paraffin for immunohistological analysis. Consecutive 4- $\mu \mathrm{m}$-thick sections were collected on gelatinised glass slides and immunofluorescent analysis was performed as described elsewhere [27, 31].
Infection of embryonic pancreases and organotypical culture Embryonic pancreatic epithelia were infected as described above for whole pancreases. After a 2-h infection period, they were washed twice with HBSS and grown in three-dimensional collagen gels as described previously $[27,28]$. Cultures were maintained for 1,3 or 7 days. The rudiments were fixed in PBS containing 4\% paraformaldehyde (Carlo-Erba Reactifs, Val de Reuil, France), cryoprotected in $15 \%$ sucrose-PBS at $4^{\circ} \mathrm{C}$ overnight, embedded in $15 \%$ sucrose $-7 \%$ gelatin PBS and frozen at $-50^{\circ} \mathrm{C}$ in isopentane (Carlo-Erba Reactifs). We collected 10- $\mu$ m-thick cryosections and analysed them by immunohistochemistry or in situ hybridisation.

Immunohistochemical procedures Immunofluorescent staining was performed according to the manufacturer's instructions. Primary antisera, applied on paraffin sections, include mouse monoclonal anti-insulin or anti-glucagon (1/2,000; Sigma-Aldrich, Saint-Quentin Fallavier, France), anti-eGFP (1/400; BD Biosciences Clontech-Ozyme, Saint Quentin Yveline, France) and rabbit polyclonal anti-insulin or anti-glucagon (1/2,000; DiaSorin, Anthony, France), antipan-cytokeratin (1/500; Dako, Trappes, France), and anticarboxypeptidase A (1/600; Biogenesis/Valbiotech AbCys, Paris, France), antibodies. The fluorescent secondary antibodies obtained from Jackson Immunoresearch Laboratories (Beckman Coulter) were fluorescein anti-mouse antibodies (1/150), fluorescein anti-rabbit antibodies $(1 / 200)$ and Texasred anti-mouse or rabbit antibodies (1/400). Photographs of the sections were taken using a fluorescence microscope (Leica, Leitz DMRB, Rueil-Malmaison, France) and digitised using a Hamamatsu (Middlesex, NJ, USA) C5810 cooled 3CCD camera.

For immunofluorescence detection on cryosections, primary antibodies used were mouse monoclonal anti-eGFP (1/400; Abcam, Cambridge, UK) and rabbit polyclonal antiinsulin $(1 / 2,000$; DiaSorin). The species-specific affinitypurified secondary antibodies included FITC-conjugated goat anti-mouse (1/150; Caltag Tebu, Burlingame, CA, USA) and CY3-conjugated goat anti-rabbit IgG (1/400; Jackson ImmunoResearch Laboratories).

Single-labelled sections incubated with mismatched secondary antibodies showed no immunostaining, confirming the specificity of the secondary antisera. Photographs of the sections were taken on a Leica, Leitz TCS confocal microscope with a krypton-argon laser beam.

Quantitative analysis Tissue sections were stained with bis benzimide Hoechst 33258 trihydrochloride (SigmaAldrich). The quantitative analysis of the number of GFPpositive and/or insulin-expressing cells per epithelium after culture was performed by counting the number of immunoreactive cells for which a nucleus was clearly detected.

Cold in situ hybridisation Cold in situ hybridisation (ISH) was performed as described previously [32, 33]. Antisense riboprobes were generated from cDNA or PCR fragments 
subcloned into the pGEMTeasy vector (Promega). The entire coding region of the eGFP fragment (711 bp) was amplified from the pTRIP $\triangle$ U3.-eGFP plasmid with the following primers: 5'-ATGGTGAGCAAGGGCGAG-3' and 5'-TACTTGTACAGCTCGTCC-3'. Both antisense and sense ngn 3 riboprobes ( $726 \mathrm{bp}$ ) were prepared as previously described [32]. Plasmids were linearised and used as templates for the synthesis of sense or antisense riboprobes by T7 or SP6 RNA polymerase (Promega), in the presence of digoxygenin-UTP (Roche Diagnostic). For double in situ hybridisation, either digoxygenin-UTP (for ngn3) or fluorescein-UTP (for eGFP) was used. Colorimetric revelations were performed with 5-bromo-4-chloro-3-indolyl phosphate and either nitroblue tetrazolium (Promega) for digoxygenin-UTP, or 2-[4-iodophenyl]-3-[4-nitrophenyl]5-phenyl-tetrazolium chloride (Roche Diagnostic) for fluorescein-UTP, to obtain the blue or red staining, respectively.

Cold in situ hybridisation and immunofluorescence double labelling ISH was performed with an ngn3 digoxygeninlabelled antisense riboprobe and revealed in blue as described above. After several washes in PBS, eGFP immunofluorescence labelling was achieved according to the manufacturer's instructions with a rabbit polyclonal anti-eGFP antibody
(1/400; BD Biosciences Clontech-Ozyme) and a fluorescein anti-rabbit secondary antibody (1/200; Jackson Immunoresearch Laboratories).

\section{Results}

Specificity of the rat insulin promoter in a lentiviral context in vitro In order to stably modify mature insulinproducing cells after gene transfer into pancreatic progenitors, we first constructed a lentiviral vector designed to restrict the transgene expression in beta cells. We produced recombinant lentiviral vectors (pTRIP $\Delta U 3$ ), pseudotyped with the VSV G-glycoprotein, that expressed the eGFP reporter gene under the control of either a 405-bp fragment of the rat insulin promoter (RIP405) or the ubiquitous CMV. To evaluate whether eGFP expression driven by the RIP405 promoter was restricted to mature beta cells in vitro, we infected Min6 cells, derived from a mouse insulinoma, and 293T cells, derived from human kidney, with either pTRIP $\Delta$ U3.RIP405-eGFP or pTRIP $\triangle$ U3.CMV-eGFP viruses. Flow cytometry analysis detected eGFP-positive cells in Min6 cells infected with pTRIP $\Delta$ U3.RIP405eGFP (Fig. 1b), but not in 293 T cells infected with the same
Fig. 1 Flow cytometry analysis of Min6 (a, b, c) and 293T (d, e, f) cells that were either untransduced $(\mathbf{a}, \mathbf{d})$ or transduced by pTRIP $\Delta$ U3.Rip405-eGFP (b, e) $\mathrm{HC}$ and pTRIP $\triangle \mathrm{U} 3 . \mathrm{CMV}$ eGFP lentiviruses $(\mathbf{c}, \mathbf{f})$. Plots of eGFP-positive cells represent cells infected with either pTRIP $\Delta$ U3.Rip405-eGFP or pTRIP

$\triangle \mathrm{U} 3 . \mathrm{CMV}$-eGFP viral vectors. Equivalent amounts of viral particles were used according to the capsid antigen p24 contents of viral stocks (200 ng of p24). Region areas selected were chosen to include eGFP-expressing cells above the autofluorescence of untransduced Min6 and $293 \mathrm{~T}$ cells as negative control. Min6 and 293T cells were infected with serial dilutions of lentiviral stocks to determine the eGFP-titres. The transduction unit $(T U)$ values indicate the number of eGFP-positive cells/ $\mathrm{ml}$ of viral stock. TU are reported below each FACS plot and were calculated as described in Materials and methods
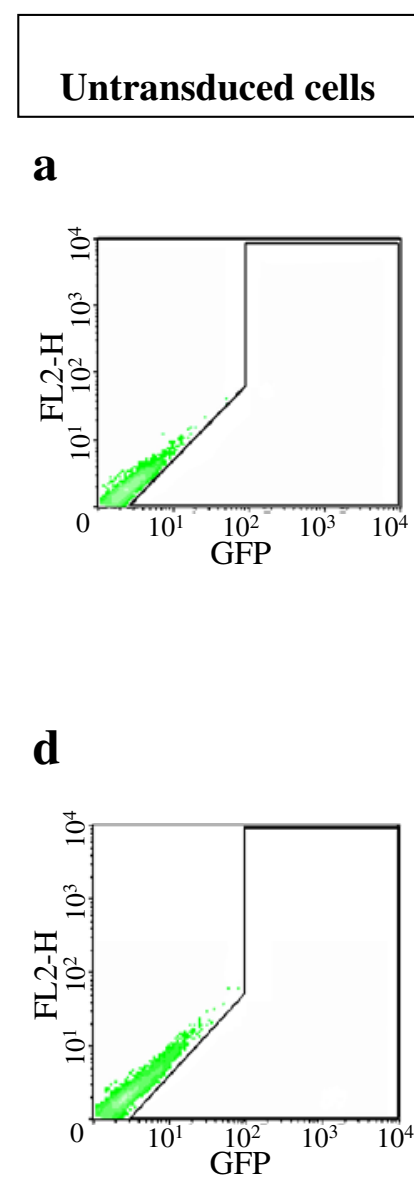

\section{pTRIP $\triangle \mathrm{U} 3$.}

\section{TU : 8.10 ${ }^{7}$ transduced cells/ml virus}

e

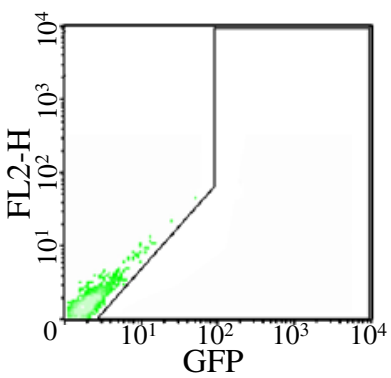

TU : 0 transduced cells/ml virus

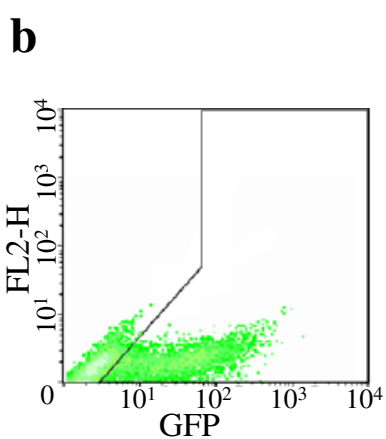

C

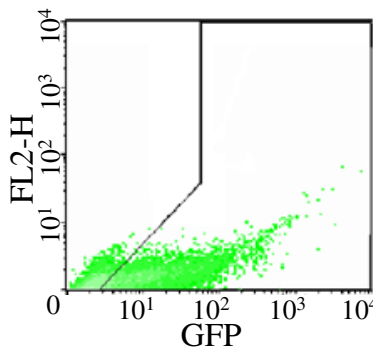

TU : $\mathbf{7 . 1 0}^{7}$ transduced cells/ml virus

f

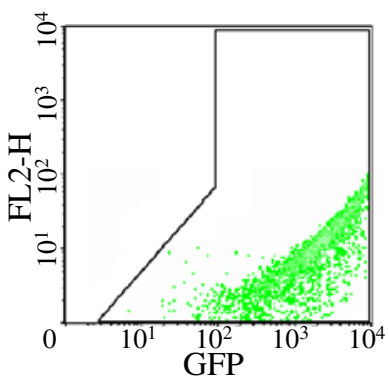

TU : $6^{6.10^{8}}$ transduced cells/ml virus 
virus (Fig. 1e). In contrast, both cell lines were transduced by the pTRIP $\Delta$ U3.CMV-eGFP (Fig. 1c, f). These results demonstrate that, in a lentiviral context, the RIP405 directs cell-specific expression in insulin-producing cells.

Development of rat embryonic pancreases engrafted in mice To determine whether stem/progenitor cells infected with lentiviral vectors are capable of producing modified mature beta cells, we generated an ex vivo model of rat beta cell differentiation. This model enabled us to investigate the ability of immature pancreatic tissue to grow and develop when engrafted into scid mice. Rat pancreatic rudiments were dissected from either 13.5 (E13.5) or 16.5 (E16.5)day-old embryos to obtain tissues containing progenitor cells at two developmental stages. Prior to the grafting of these pancreases, we characterised the presence of endocrine and exocrine tissue in the explant by immunofluorescence analysis. In accordance with previously reported results [34], at E16.5 the vast majority of the endocrine cells in the pancreas are glucagon-expressing cells, in addition to rare insulin-expressing cells (Fig. 2a). Moreover, carboxypeptidase A (CPA)-expressing cells were found dispersed in the tissue and did not display typical acinar morphology (Fig. 2c). E16.5 pancreases were transplanted under the kidney capsule of scid mice and analysed 7 days later (Fig. 2b, d). During this period, numerous CPAexpressing cells developed and associated to form acinar structures (Fig. 2d). Under these conditions, insulin-expressing cells also developed and were found associated with glucagon-expressing cells, forming clusters with a similar morphology to that of islets of Langherhans (Fig. 2b). This increase in the number of differentiated mature beta cells was accompanied by a 250 -fold increase in insulin content of the graft as measured by radioimmunoassay (data not shown).

The increase in beta cell mass during the transplantation period could be due either to the proliferation of the rare existing insulin-expressing cells, or to the differentiation of progenitor cells into mature beta cells. To discern between the two possibilities, we thus repeated the transplantation experiments with E13.5 pancreases, which do not contain beta cells at this stage (Fig. 2e), whereas, glucagon-expressing cells (Fig. 2e) or CPA-containing cells (Fig. 2g) are barely present. Transplantation of these pancreases was performed as previously described and analysed 7 days later (Fig. 2f, h). Exocrine tissue developed as revealed by the detection of CPA-expressing cells with normal acinar morphology (Fig. 2h). In addition, we observed insulinand glucagon-expressing cells forming structures resembling islets of Langerhans (Fig. 2f). These results clearly indicate that the increase of the beta cell mass observed in our model is mainly due to the differentiation and not the proliferation of pre-existing beta cells.

Together, these data demonstrate that we generated a functional ex vivo model for rat pancreatic development. When engrafted under the kidney capsule of scid mice, E13.5 and E16.5 immature pancreases develop into endocrine and exocrine tissues with normal morphology.

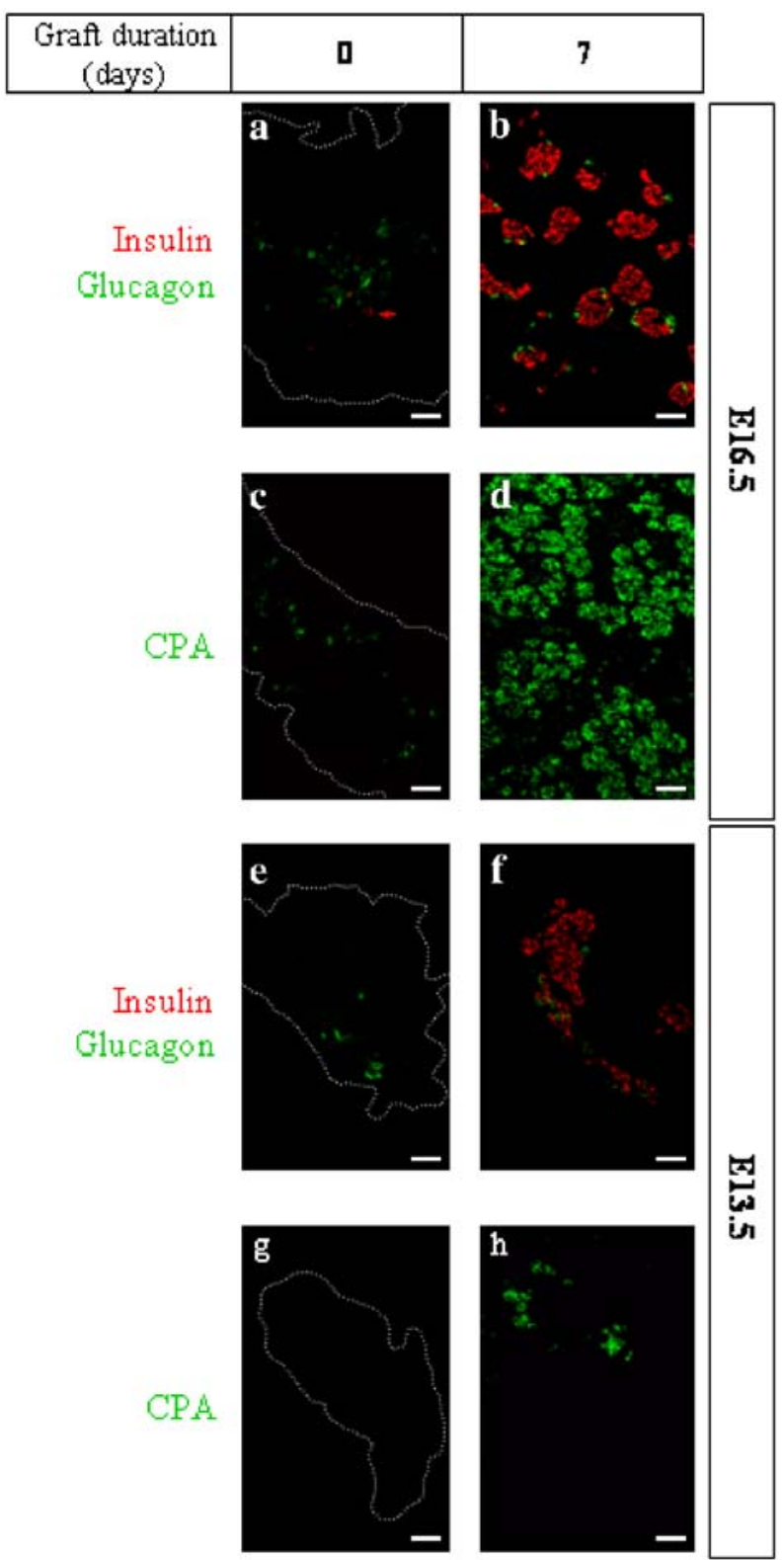

Fig. 2 Development of rat embryonic pancreases engrafted in scid mice. Endocrine cells in E16.5 pancreases were mainly glucagonpositive cells (green) with rare dispersed insulin-positive cells (red; red arrow) (a). A few exocrine cells were detectable by CPA immunostaining (green), but did not form acinar structures (c). Seven days after transplantation, numerous insulin-positive cells were detected (red) associated with glucagon-expressing cells (green) to form islets of Langerhans (b). CPA-positive cells (green) form clusters similar to acini (d). At E13.5, no insulin or CPA-expressing cells were detected (e, $\mathbf{g})$ before grafting. Rare glucagon-positive cells (green) were present and dispersed (e). Seven days after transplantation, endocrine insulin (f) (red), glucagon (green) and exocrine (CPA, green $)(\mathbf{h})$ cells were detected with a morphology similar to that of islets of Langerhans or acini. The contours of the pancreatic rudiments are indicated as a dotted line. Scale bar $50 \mu \mathrm{m}$

Transduction with recombinant lentiviruses mediates longterm expression of cell-type-specific eGFP expression in insulin-positive cells In our in vivo model, we sought to determine whether recombinant lentiviruses can infect 
progenitor cells that will differentiate into beta cells specifically expressing the transgene. E13.5 and E16.5 pancreases were infected with the pTRIP $\Delta$ U3.RIP405-eGFP vector, grafted and analysed 7 days later. Under these conditions, no eGFP-expressing cells were detected (data not shown).

We next infected E16.5 immature pancreases that were partially dissociated prior to infection and transplantation. We reasoned that the dissociation of the pancreas would increase the accessibility of the viral particles to the epithelium and thus allow eGFP expression. Using this technique, eGFP expression was observed in insulin-containing cells (Fig. 3a).

To define the cell types expressing eGFP in the infected pancreases that developed in the scid mice, double immuno-

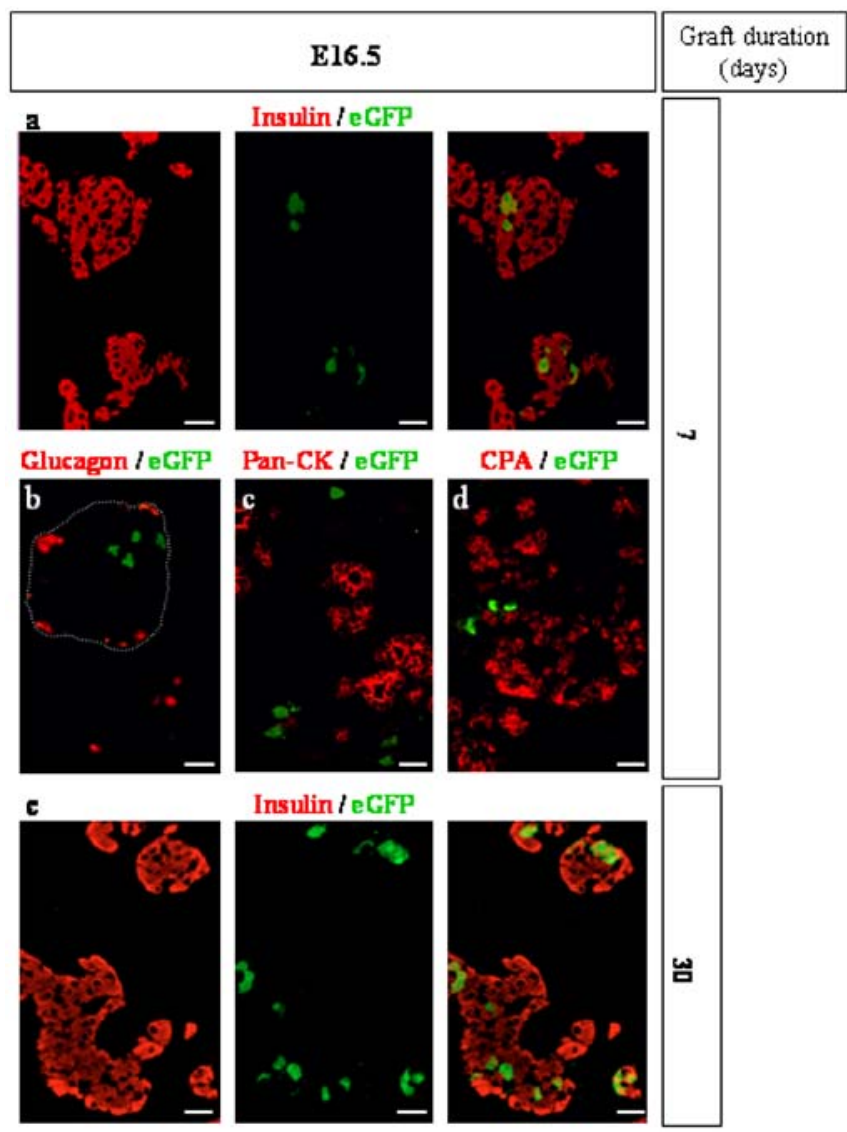

Fig. 3 Restricted expression of eGFP in insulin-positive cells and long-term expression after infection with recombinant lentiviruses. E16.5 pancreases were partially dissociated, infected with lentiviruses expressing eGFP under the control of the insulin promoter (pTrip $\Delta$ U3.RIP405-eGFP) (120 ng of p24), grafted in scid mice and analysed 7 (a, b, c, d) or 30 (e) days after transplantation. a, e Immunodetection of insulin (red, left panel), eGFP (green, middle panel) and merge of both stainings (right panel). All eGFP-positive cells were insulin-positive 7 or 30 days after transplantation. b Immunodetection of glucagon (red) and eGFP (green). The contour of an islet of Langerhans is represented as a dotted line. $\mathbf{c}$ Immunodetection of pan-cytokeratin (red) and eGFP (green). d Immunodetection of CPA (red) and eGFP (green). Note that the eGFP staining was found in nuclear and cytoplasmic compartments. No eGFP-positive cells were identified as alpha cells, duct cells or acinar cells $(\mathbf{b}, \mathbf{c}, \mathbf{d})$. Scale bar $50 \mu \mathrm{m}$ labelling was performed using antibodies directed against insulin (beta cells), glucagon (alpha cells), pan-cytokeratin (duct cells), or carboxypeptidase-A (acinar cells) in combination with anti-eGFP. As shown in Fig. 3a, 7 days after transplantation eGFP was clearly detected in insulin-expressing cells, while eGFP was never detected in glucagonexpressing cells (Fig. 3b), pan-cytokeratin-expressing cells (Fig. 3c) or CPA-expressing cells (Fig. 3d). We also analysed the grafts 1 month after transplantation. As shown in Fig. 3e, sustained beta cell expression of eGFP was achieved.

To rule out the possibility that the eGFP-labelled insulinexpressing cells originated from the rare insulin-expressing cells present before transplantation in E16.5 pancreases (Fig. 2a), we used E13.5 pancreases, which lack these preexisting beta cells (Fig. 2e). As shown in Fig. 4, 7 days after transplantation, eGFP was present in beta cells that have clearly differentiated in this model.

Highly efficient lentiviral transduction of immature pancreatic rudiments As described above, the pTRIP $\Delta \mathrm{U} 3$. RIP405-eGFP vector transduces E13.5 and E16.5 pancreatic rudiments, with eGFP transgene expression being restricted to insulin-producing cells. However, the transduction efficiency was less than $5 \%$ of the insulin-containing cells.

To improve the transduction efficiency, we used an organotypical culture model of E13.5 rat pancreatic epithelium, where both the accessibility of the viral particles to the epithelium and endocrine cell differentiation are favoured. At E13.5 in rats, the pancreatic primordium consists of a central epithelial bud surrounded by a considerable amount of mesenchymal tissue. We and others previously demonstrated that the mesenchymal tissue is not necessary for the differentiation of the epithelium into beta cells [26, 27]. Therefore, the mesenchymal tissue was removed. The isolated pancreatic epithelium was infected with the lentiviral vectors, pTRIP $\Delta \mathrm{U} 3$.CMV-eGFP and pTRIP $\Delta \mathrm{U} 3$. RIP405-eGFP for $2 \mathrm{~h}$ and cultured in conditions that

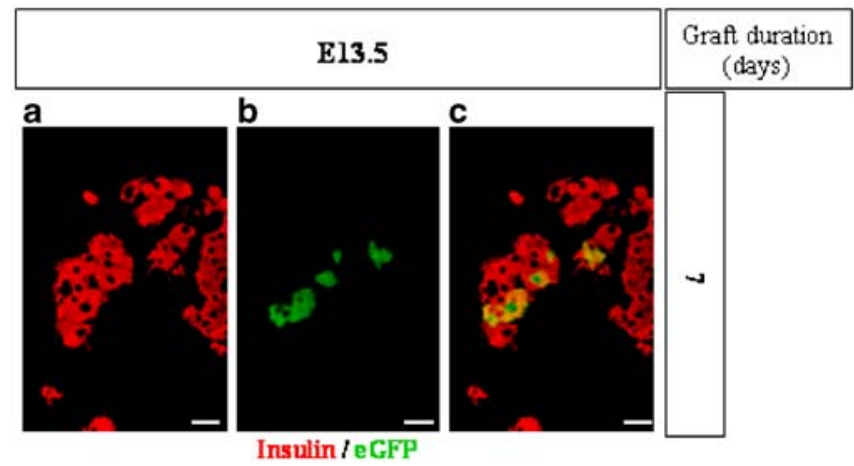

Fig. 4 Restricted expression of eGFP in insulin-positive cells after infection of immature pancreases that lack beta cells. E13.5 pancreases that lacked insulin-expressing cells were partially dissociated, infected with pTrip $\Delta$ U3.RIP405-eGFP (120 ng of p24) and grafted to scid mice. Seven days after transplantation, numerous insulinpositive cells (red) (a) that had differentiated were eGFP-positive (b). c The merged images from $\mathbf{a}$ and $\mathbf{b}$. Note that the eGFP staining was found both in nuclear and cytoplasmic compartments, while insulin staining was restricted to the cytoplasmic compartment. Scale bar $50 \mu \mathrm{m}$ 
simulate pancreatic differentiation [27, 35]. To evaluate the transduction yield of both vectors, double immunofluorescence detection of insulin and eGFP was performed after 3 and 7 days of culture. We observed insulin-expressing cells after 3 days in culture (Fig. 5a, c) and a further increase in the number of differentiated beta cells was detected after 7 days (Fig. 5b, d). In explants infected with pTRIP $\Delta$ U3.RIP405-eGFP vector, we observed that all of the eGFP-expressing cells stained positive for insulin (Fig. 5a, b; Fig. 6a, b left panel). Moreover, the variation of eGFP expression during the culture period was concomitant and parallel to that observed for insulin. The ratio of

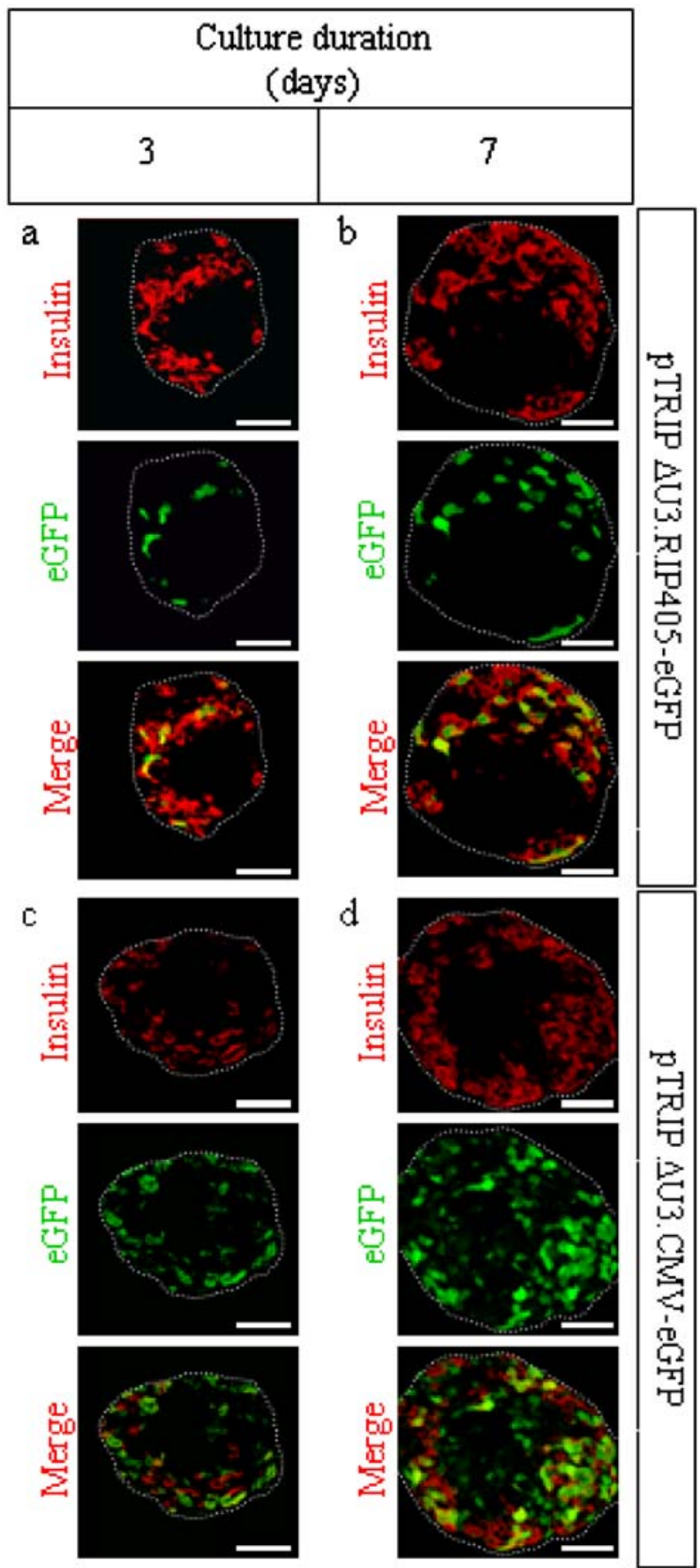

insulin-positive cells versus the total number of cells increased from 20.2 to $46.0 \%$ between day 3 and day 7 of culture (Fig. 6b, left panel), and a similar variation of 9.7$32.4 \%$ was observed for the percentage of eGFP-expressing cells relative to the total number of cells (Fig. 6a, left panel). Finally, the transduction yield of insulin-positive cells was highly efficient, since 7 days after infection $70.4 \%$ of insulin-expressing cells co-expressed eGFP (Fig. 6b, left panel).

The explants were infected in parallel with viruses where eGFP transcription was under the control of the CMV promoter and cultured for 3 or 7 days. As shown in Fig. 5c and $\mathrm{d}$, a large number of eGFP-expressing cells that stained either positive or negative for insulin were observed, reflecting the ubiquitous expression feature of the CMV promoter. In addition, the percentage of eGFP-positive cells relative to the total number of cells was not significantly different after 3 or 7 days in culture (33.8 and $41.3 \%$, respectively; Fig. 6 b, right panel). This demonstrates that in our organotypical culture model, the ubiquitous CMV promoter drives the expression of a transgene in a manner independent of the differentiation status.

Beta cells expressing eGFP derive from infected progenitors To further demonstrate that lentiviral vectors could target endocrine progenitors, E13.5 pancreatic epithelia were infected with the pTRIP $\triangle \mathrm{U} 3 . \mathrm{CMV}$-eGFP lentiviral vector. After $24 \mathrm{~h}$ in culture, both double in situ hybridisation and in situ hybridisation coupled with immunofluoescence labelling were performed to delineate whether a portion of the eGFP-positive cells co-expressed ngn3, a marker of endocrine progenitor cells [36, 37]. First we addressed the specificity of ngn3 detection by in situ hybridisation. As observed in Fig. 7a, the use of an antisense ngn3 riboprobe resulted in the detection of clearly labelled cells whereas no signal was observed with the sense ngn3 riboprobe (Fig. 7b). We next performed double in situ hydridisation where the eGFP mRNA was first revealed in red (Fig. 7c) and the ngn 3 mRNA was subsequently visualised in blue on the same section (Fig. 7d). Our results clearly illustrate that numerous eGFP-expressing cells are indeed ngn3 progenitor cells. Similar results were obtained with a different method, in which eGFP immunofluorescence de-

Fig. 5 High-efficiency lentiviral transduction of immature pancreatic rudiments that developed in culture. E13.5 pancreatic rudiments were depleted of their surrounding mesenchyme, infected with either pTRIP $\Delta$ U3.RIP405-eGFP (85 ng of p24), or pTRIP $\Delta$ U3.CMVeGFP (120 ng of p24), and cultured in a collagen matrix. Confocal dual immunofluorescence detection was performed on both insulin (red) and eGFP (green) after 3 or 7 days in culture on $10-\mu \mathrm{m}$ cryosections. After 3 days in culture, insulin-positive cells were detected (a, c). A marked increase in the number of insulin-expressing cells was observed after 7 days in culture $(\mathbf{b}, \mathbf{d})$. Upon infection with the pTRIP $\triangle$ U3.RIP405-eGFP, the eGFP and insulin expression kinetics were similar; all eGFP-expressing cells stained positive for insulin $(\mathbf{a}, \mathbf{b})$. The use of a ubiquitous promoter (pTrip $\triangle$ U3.CMVeGFP) allowed efficient eGFP expression after 3 (c) or 7 (d) days in culture. Numerous eGFP-positive cells $(\mathbf{c}, \mathbf{d})$ were either insulinpositive or negative. The contours of the pancreatic explants are indicated as a dotted line. Scale bar $50 \mu \mathrm{m}$ 


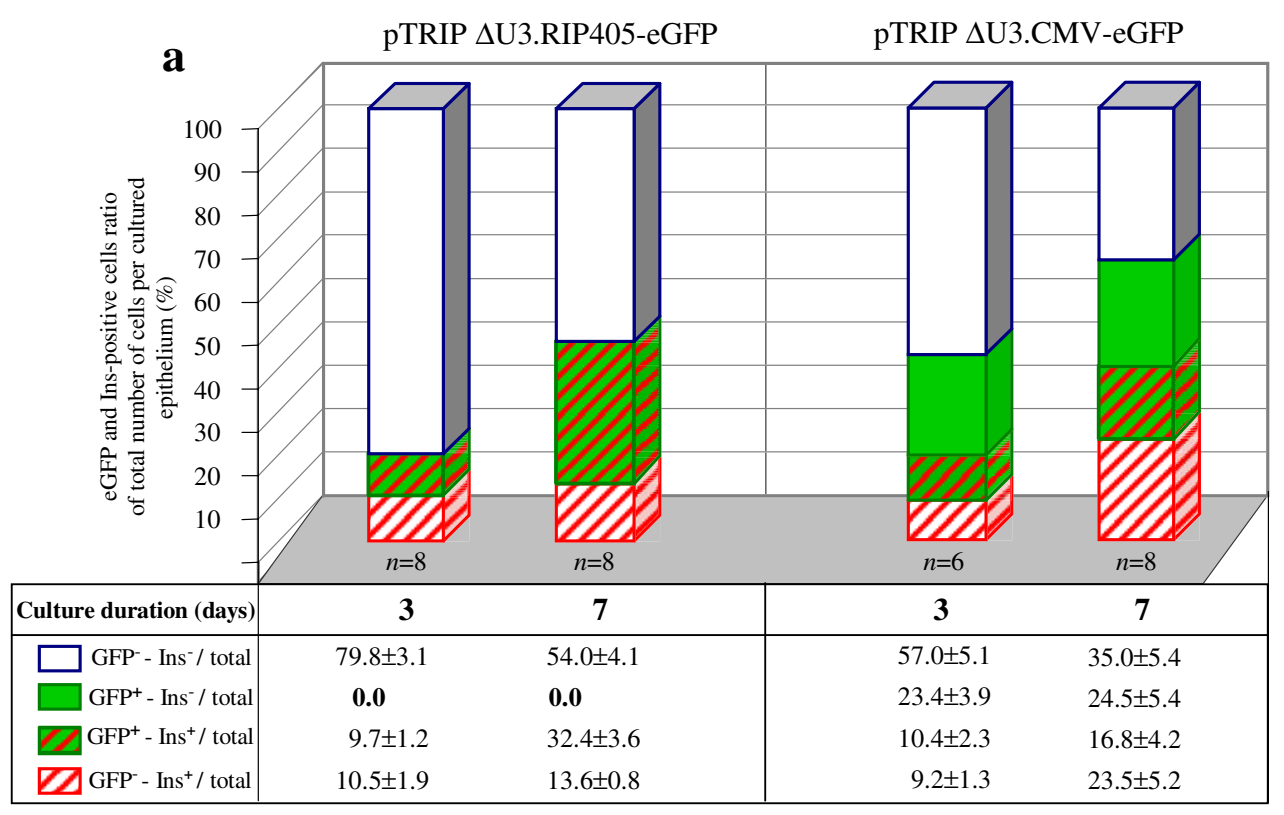

\begin{tabular}{|c|c|c|c|c|c|c|c|}
\hline \multirow[t]{2}{*}{ b } & \multicolumn{2}{|c|}{$\begin{array}{c}\text { Culture } \\
\text { duration (days) }\end{array}$} & \multirow{2}{*}{ 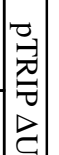 } & & \multicolumn{2}{|c|}{$\begin{array}{c}\text { Culture } \\
\text { duration (days) }\end{array}$} & \multirow{2}{*}{ 吾 } \\
\hline & 3 & 7 & & & 3 & 7 & \\
\hline $\mathrm{Ins}^{+} /$total $(\%)$ & $20.2 \pm 3.1 *$ & $46.0 \pm 4.1 * *$ & 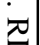 & Ins + total (\%) & $19.6 \pm 3.5^{*}$ & $40.4 \pm 3.1 * *$ & $\vec{\omega}$ \\
\hline $\begin{array}{l}\mathrm{GFP}^{+}-\mathrm{Ins}^{+} / \mathrm{Ins}^{+}(\%) \\
\mathrm{GFP}^{+}-\mathrm{Ins} / \text { total }(\%)\end{array}$ & $\begin{array}{c}48.2 \pm 1.0^{\circ \mathrm{S}} \\
0\end{array}$ & $\begin{array}{c}70.4 \pm 2.0^{\circ \mathrm{S}} \\
0\end{array}$ & 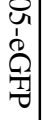 & $\mathrm{GFP}^{+} /$total $(\%)$ & $33.8 \pm 4.7 * * *$ & $41.3 \pm 4.7 * * *$ & 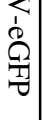 \\
\hline
\end{tabular}

Fig. 6 Quantitative analysis of transduction yield. E13.5 depleted pancreatic epithelia were infected with either pTRIP $\Delta$ U3.RIP405eGFP (85 ng of p24) or pTRIP $\triangle$ U3.CMV-eGFP (120 ng of p24) viruses and cultured for 3 or 7 days. The epithelia for each experimental culture condition were sectioned and the total number of cells was counted after nucleus staining. Dual immunofluorescence analysis was performed on the sections to visualise eGFP- and insulin-positive cells. The merge of both fluorescent markers revealed four different types of cells and the number of cells in each population was determined. a The diagram displays the percentage of each population relative to the total number of cells. The used values are presented in a table below the diagram and represent means \pm SEM. $n$ represents the number of independent infected epithelia used for each condition. b Mean values displayed a Gaussian

tection was achieved after ngn3 in situ hybridisation staining (Fig. 7e-g).

\section{Discussion}

In this study, we have demonstrated that recombinant lentiviral vectors can efficiently infect pancreatic progenitor cells and thereby stably modify mature rat pancreatic beta cells.

During the past years, different approaches have been used to genetically modify beta cells with two major objectives: beta cell protection against immune destruction; distribution, thus allowing Student's $t$-test statistical analysis. Pairwise comparisons were performed. Insulin development after $3\left({ }^{*} p=0.448\right)$ or $7(* *=0.155)$ days in culture was not significantly different between the two types of viral vectors. After infection with the pTRIP $\triangle U$ U.CMV-eGFP virus, the relative amount of eGFP-positive cells versus the total number of cells $(* * * p=0.148)$ was not significantly different after 3 or 7 days in culture, demonstrating that the ubiquitous CMV promoter drives the expression of the eGFP transgene in a manner independent of the differentiation status. Finally, the observed increase of the proportion of insulin-positive cells that co-expressed eGFP between 3 and 7 days in culture after infection with pTRIP $\Delta$ U3.RIP405-eGFP was significantly different $\left({ }^{\circ \mathrm{S}}, p<0.001\right)$

and beta cell expansion. For these purposes, recombinant DNA was transferred into mature beta cells using different vectors, such as adenoviruses, adeno-associated viruses and lentiviruses [38]. While progress has been made in ways of protecting beta cells against immune destruction [10], attempts to expand beta cells by gene transfer directly into insulin-producing cells have been unsuccessful. For example, beta cell lines generated by infection with retroviral vectors expressing the SV40 large T antigen, H-RAS and hTERT oncogenes lose insulin expression with time, suggesting that the cells de-differentiate [39]. Beta cell differentiation could be reactivated by transfection of specific transcription factors combined with the use of specific 


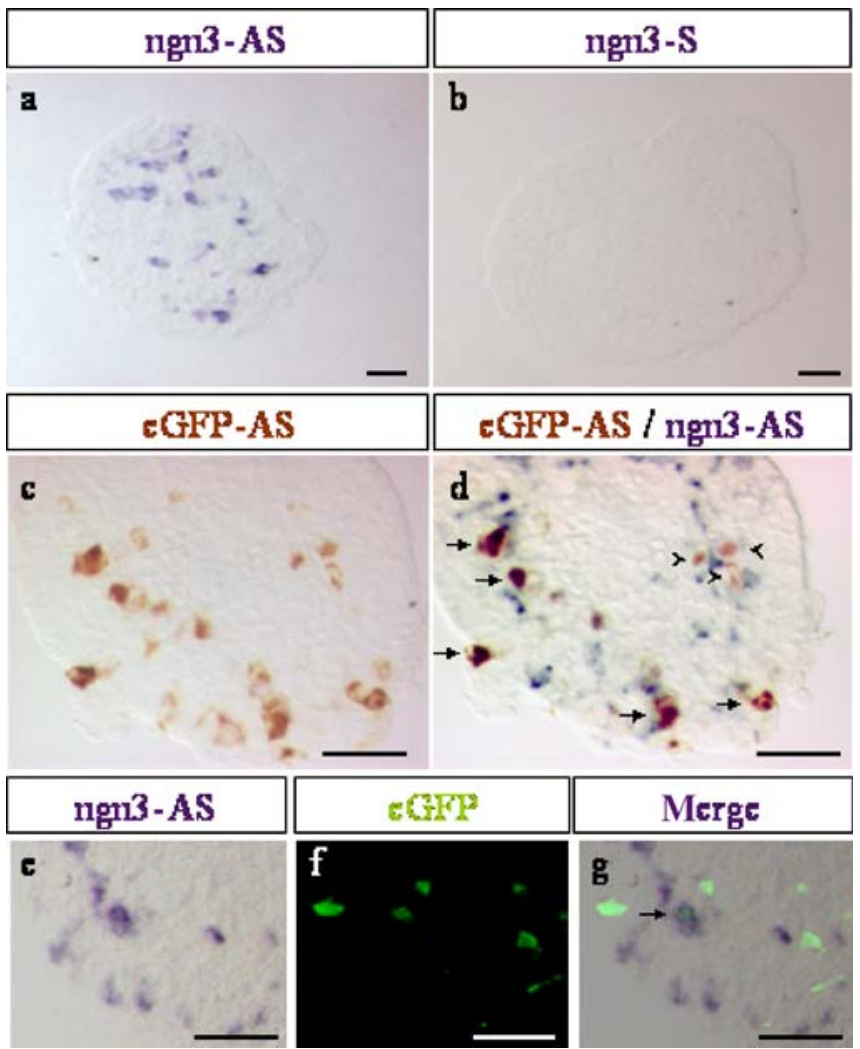

Fig. 7 Neurogenin 3 endocrine progenitor cells can be transduced by recombinant lentiviruses. E13.5 pancreatic rudiments were depleted of their surrounding mesenchyme, infected with pTRIP $\Delta U 3$. CMV-eGFP (120 ng of p24), and cultured for $24 \mathrm{~h}$ in a collagen matrix. Single in situ hybridisation was performed on $10-\mu \mathrm{m}$ cryosections to detect neurogenin 3 (ngn3) expression. The specificity of ngn3 detection was addressed by comparing in situ hybridisation labelling with either antisense (a) (ngn3-AS) or sense (b) (ngn3-S) ngn3 riboprobes. Double in situ hybridisation was performed allowing dual detection of eGFP and ngn3. The eGFP staining was first visualised (c) prior to ngn3 labelling (d). Three types of labelled cells (d) were observed: ngn3 single-labelled cells (blue); eGFP singlelabelled cells (black arrowheads); and eGFP/ngn3 double-labelled cells (black arrows). Note that when double in situ hybridisation was performed using eGFP-AS and ngn3-S probes, no ngn3 blue staining was observed (data not shown). e- $\mathbf{g}$ Immunofluorescence detection of eGFP was achieved after ngn3 labelling by in situ hybridisation. These panels show e ngn3 in situ hybridisation staining, $\mathbf{f}$ eGFP immunofluorescence labelling, and $\mathbf{g}$ the merged images. The black arrow (g) points to an eGFP/ngn3 double-labelled cell. Scale bar $50 \mu \mathrm{m}$

growth factors. However, even in such conditions, the levels of insulin expression remained low when compared to mature beta cells [40]. Other investigators were unable to stably immortalise beta cells using lentiviruses expressing the SV40 T antigen [11]. Although gene transfer was efficient, the inability to obtain an immortalised cell line may be due to the use of mature beta cells as infected target cells. To circumvent this limitation, we have addressed here the use of lentiviral vectors as a tool to stably transduce pancreatic endocrine progenitor cells, instead of mature beta cells.

We designed an ex vivo transplantation experimental model in which progenitor cells differentiate into beta cells.
We grafted embryonic pancreases under the kidney capsule of immuno-incompetent mice. This grafting site has been shown to permit the development of a large number of tissues, such as rodent thymus, bovine adrenocortical cells and human embryonic and fetal pancreases [29, 30, 40, 41]. Within 1 week after transplantation of E16.5 pancreases, the insulin content was multiplied by a factor of 250 . Such a beta cell increase could not be due to the proliferation of the few pre-existing insulin-expressing cells for the following reasons. First, beta cell proliferation is very limited during prenatal development [42]. Second, it is now clearly established that the rare insulin-expressing cells present during development of the embryonic pancreas and which frequently co-express glucagon are not the progenitors of mature beta cells [42, 43]. Lastly, E13.5 pancreases that do not contain beta cells develop into beta cells when grafted to scid mice (the present work). Thus, our data indicate that the observed development of endocrine tissue in our ex vivo experimental model is due to the differentiation of progenitor cells originally present in the transplanted tissue.

In order to infect the pancreatic progenitor cells and subsequently transduce mature beta cells, access of the virus to the progenitor cell population is a crucial experimental step. There are different arguments indicating that pancreatic progenitor cells are located in the centre of the pancreatic tissue: (1) during embryonic life, the cells expressing ngn3, a marker of endocrine pancreatic progenitor cells, are located in the central region of the developing pancreas that corresponds to the pancreatic epithelium [36, 44-46]; (2) the first insulin-expressing cells also appear in the central region of the pancreas [47]. However, it is well established that in compact tissues, the accessibility of viral particles to cells located in the centre of the tissue is poor [3]. We show here that by partially dissociating pancreases, we increase the probability of contact between the virus and the progenitor cells and demonstrate that progenitor cells that differentiate into beta cells can indeed be infected. The infection yield of progenitors was further enhanced by direct exposure of purified embryonic pancreatic epithelium to the viruses upon removal of the surrounding mesenchyme.

As discussed above, our data indicate that the beta cells expressing eGFP derived from the infection of progenitor cells that differentiate into beta cells rather than from the infection of pre-existing beta cells that proliferate. It was, therefore, of crucial importance to characterise the nature of the cells that were the primary cell target infected by the lentiviral vectors. We showed that numerous ngn3-positive cells expressed eGFP $24 \mathrm{~h}$ after infection with a lentiviral vector, in which eGFP expression was under the control of the ubiquitous CMV promoter. This demonstrates that endocrine progenitor cells can be infected and a restricted transgene expression obtained according to the specificity of the promoter.

The experimental process described here represents a powerful tool for gain-of-function experiments in a dynamic developmental model that can allow promoter-specific targeting throughout the entire cell differentiation cascade in the pancreas. In addition, our model is also appropriate for loss-of-function studies, since recent data have shown that 
RNA interference can be efficiently performed using lentiviral vectors [48-50].

We designed, in this study, a new model that resembles those used in vivo to produce rodent beta cell lines. In these cases, insulinomas were produced from transgenic mice expressing an oncogene under the control of the insulin promoter allowing the expression of the transgene specifically in mature beta cells $[24,51]$. This approach was used to generate rodent beta cell lines that were functional in terms of insulin secretion upon glucose simulation. However, the beta cell lines were obtained by gene transfer in fertilised eggs, which restricts its application to animal models without any possible transfer to humans. The model presented here is based on ex vivo gene transfer, which is applicable to both animal and human tissues. Recently, we designed an ex vivo system, where human embryonic pancreases can develop into mature pancreatic tissues [29]. Such a model will now be used to test the feasibility of infecting human pancreatic progenitor cells with a specific expression of the transgene in mature human beta cells.

Acknowledgements The first two authors contributed equally to this work. This work was supported by the Juvenile Diabetes Research Foundation (JDRF Center for Beta Cell Therapy in Europe), INSERM/Fondation pour la Recherche Médicale/Juvenile Diabetes Research Foundation (4DA03H), the Association Francaise des Diabétiques (AFD), the Fondation pour la Recherche Médicale, INSERM, the region Ile de France, Retina France and the University Paris VI.

The authors are deeply grateful to Yasmine Hazhouz, Emilie Neveu, Isabelle LeNin and Stephanie Bauchet for technical assistance and to Medeva Ghee and Chamsy Sarkis for helpful comments and critical reading of the manuscript. Muriel Castaing was successively supported by the Foundation pour la Recherche Medicale (FRM) and by l'Association d'Aide aux Jeunes Diabétiques (AJD). Aline Guerci was successively supported by the Ministère de la Recherche et de la Technologie and by the FRM.

\section{References}

1. Shapiro AM, Lakey JR, Ryan EA et al (2000) Islet transplantation in seven patients with type 1 diabetes mellitus using a glucocorticoid-free immunosuppressive regimen. N Engl J Med 343:230-238

2. Halban PA, Kahn SE, Lernmark A, Rhodes CJ (2001) Gene and cell-replacement therapy in the treatment of type 1 diabetes: how high must the standards be set? Diabetes 50:2181-2191

3. Leibowitz G, Beattie GM, Kafri T et al (1999) Gene transfer to human pancreatic endocrine cells using viral vectors. Diabetes 48:745-753

4. Csete ME, Benhamou PY, Drazan KE et al (1995) Efficient gene transfer to pancreatic islets mediated by adenoviral vectors. Transplantation 59:263-268

5. Becker TC, BeltrandelRio H, Noel RJ, Johnson JH, Newgard CB (1994) Overexpression of hexokinase I in isolated islets of Langerhans via recombinant adenovirus. Enhancement of glucose metabolism and insulin secretion at basal but not stimulatory glucose levels. J Biol Chem 269:21234-21238

6. Verma IM, Somia N (1997) Gene therapy—promises, problems and prospects. Nature 389:239-242

7. Kapturczak M, Zolotukhin S, Cross J et al (2002) Transduction of human and mouse pancreatic islet cells using a bicistronic recombinant adeno-associated viral vector. Mol Ther 5:154160
8. Flotte T, Agarwal A, Wang J et al (2001) Efficient ex vivo transduction of pancreatic islet cells with recombinant adenoassociated virus vectors. Diabetes 50:515-520

9. Ju Q, Edelstein D, Brendel MD et al (1998) Transduction of non-dividing adult human pancreatic beta cells by an integrating lentiviral vector. Diabetologia 41:736-739

10. Gallichan WS, Kafri T, Krahl T, Verma IM, Sarvetnick N (1998) Lentivirus-mediated transduction of islet grafts with interleukin 4 results in sustained gene expression and protection from insulitis. Hum Gene Ther 9:2717-2726

11. Salmon P, Oberholzer J, Occhiodoro T, Morel P, Lou J, Trono D (2000) Reversible immortalization of human primary cells by lentivector-mediated transfer of specific genes. Mol Ther 2 : 404-414

12. Uchida N, Buck DW, He D et al (2000) Direct isolation of human central nervous system stem cells. Proc Natl Acad Sci U S A 97:14720-14725

13. Case SS, Price MA, Jordan CT et al (1999) Stable transduction of quiescent CD34(+)CD38(-) human hematopoietic cells by HIV-1-based lentiviral vectors. Proc Natl Acad Sci U S A 96: 2988-2993

14. Miyoshi H, Smith KA, Mosier DE, Verma IM, Torbett BE (1999) Transduction of human CD34+ cells that mediate longterm engraftment of NOD/SCID mice by HIV vectors. Science 283:682-686

15. Evans JT, Kelly PF, O’Neill E, Garcia JV (1999) Human cord blood CD34+CD38-cell transduction via lentivirus-based gene transfer vectors. Hum Gene Ther 10:1479-1489

16. Pawliuk R, Westerman KA, Fabry ME et al (2001) Correction of sickle cell disease in transgenic mouse models by gene therapy. Science 294:2368-2371

17. Buchet D, Serguera C, Zennou V, Charneau P, Mallet J (2002) Long-term expression of beta-glucuronidase by genetically modified human neural progenitor cells grafted into the mouse central nervous system. Mol Cell Neurosci 19:389-401

18. Flax JD, Aurora S, Yang C et al (1998) Engraftable human neural stem cells respond to developmental cues, replace neurons, and express foreign genes. Nat Biotechnol 16:1033-1039

19. Englund U, Ericson C, Rosenblad C et al (2000) The use of a recombinant lentiviral vector for ex vivo gene transfer into the rat CNS. NeuroReport 11:3973-3977

20. Zennou V, Petit C, Guetard D, Nerhbass U, Montagnier L, Charneau P (2000) HIV-1 genome nuclear import is mediated by a central DNA flap. Cell 101:173-185

21. Sanvito F, Nichols A, Herrera $P$ et al (1995) TGF- $\beta 1$ overexpression in murine pancreas induces chronic pancreatitis and, together with TNF- $\alpha$, triggers insulin-dependent diabetes. Biochem Biophys Res Commun 217:1279-1286

22. Zufferey R, Nagy D, Mandel RJ, Naldini L, Trono D (1997) Multiply attenuated lentiviral vector achieves efficient gene delivery in vivo. Nat Biotechnol 15:871-875

23. Yee JK, Miyanohara A, LaPorte P, Bouic K, Burns JC, Friedmann T (1994) A general method for the generation of high-titer, pantropic retroviral vectors: highly efficient infection of primary hepatocytes. Proc Natl Acad Sci U S A 91:95649568

24. Miyazaki J, Araki K, Yamato E et al (1990) Establishment of a pancreatic beta cell line that retains glucose-inducible insulin secretion: special reference to expression of glucose transporter isoforms. Endocrinology 127:126-132

25. Gregory LG, Harbottle RP, Lawrence L, Knapton HJ, Themis M, Coutelle C (2003) Enhancement of adenovirus-mediated gene transfer to the airways by DEAE dextran and sodium caprate in vivo. Mol Ther 7:19-26

26. Gittes G, Galante P, Hanahan D, Rutter W, Debas H (1996) Lineage specific morphogenesis in the developing pancreas: role of mesenchymal factors. Development 122:439-447

27. Miralles F, Czernichow P, Scharfmann R (1998) Follistatin regulates the relative proportions of endocrine versus exocrine tissue during pancreatic development. Development 125:10171024 
28. Rachdi L, Marie JC, Scharfmann R (2003) Role for VPAC2 receptor-mediated signals in pancreas development. Diabetes 52:85-92

29. Castaing M, Peault B, Basmaciogullari A, Casal I, Czernichow P, Scharfmann R (2001) Blood glucose normalization upon transplantation of human embryonic pancreas into beta-celldeficient SCID mice. Diabetologia 44:2066-2076

30. Thomas M, Northrup SR, Hornsby PJ (1997) Adrenocortical tissue formed by transplantation of normal clones of bovine adrenocortical cells in scid mice replaces the essential functions of the animals' adrenal glands. Nat Med 3:978-983

31. Cras-Meneur C, Elghazi L, Czernichow P, Scharfmann R (2001) Epidermal growth factor increases undifferentiated pancreatic embryonic cells in vitro: a balance between proliferation and differentiation. Diabetes 50:1571-1579

32. Ravassard P, Chatail F, Mallet J, Icard-Liepkalns C (1997) Relax, a novel rat bHLH transcriptional regulator transiently expressed in the ventricular proliferating zone of the developing central nervous system. J Neurosci Res 48:146-158

33. Herzog E, Bellenchi GC, Gras C et al (2001) The existence of a second vesicular glutamate transporter specifies subpopulations of glutamatergic neurons. J Neurosci 21:RC181

34. Rall LB, Pictet RL, Williams RH, Rutter WJ (1973) Early differentiation of glucagon-producing cells in embryonic pancreas: a possible developmental role for glucagon. Proc Natl Acad Sci U S A 70:3478-3482

35. Miralles F, Serup P, Cluzeaud F, Vandewalle A, Czernichow P, Scharfmann R (1999) Characterization of beta cells developed in vitro from rat embryonic pancreatic epithelium. Dev Dyn 214: $116-126$

36. Gradwohl G, Dierich A, LeMeur M, Guillemot F (2000) Neurogenin 3 is required for the development of the four endocrine cell lineages of the pancreas. Proc Natl Acad Sci U S A 97:16071611

37. Gu G, Dubauskaite J, Melton DA (2002) Direct evidence for the pancreatic lineage: NGN3+ cells are islet progenitors and are distinct from duct progenitors. Development 129:24472457

38. Demeterco C, Levine F (2001) Gene therapy for diabetes. Front Biosci 6:D175-D191
39. De la Tour D, Halvorsen T, Demeterco C et al (2001) Beta-cell differentiation from a human pancreatic cell line in vitro and in vivo. Mol Endocrinol 15:476-483

40. Hoffmann MW, Allison J, Miller JF (1992) Tolerance induction by thymic medullary epithelium. Proc Natl Acad Sci U S A $89: 2526-2530$

41. Hayek A, Beattie GM (1997) Experimental transplantation of human fetal and adult pancreatic islets. J Clin Endocrinol Metab 82:2471-2475

42. Jensen J, Heller RS, Funder-Nielsen T et al (2000) Independent development of pancreatic alpha- and beta-cells from neurogenin3-expressing precursors: a role for the notch pathway in repression of premature differentiation. Diabetes 49:163-176

43. Herrera PL (2000) Adult insulin- and glucagon-producing cells differentiate from two independent cell lineages. Development 127:2317-2322

44. Gu G, Brown JR, Melton DA (2003) Direct lineage tracing reveals the ontogeny of pancreatic cell fates during mouse embryogenesis. Mech Dev 120:35-43

45. Apelqvist A, Li H, Sommer L et al (1999) Notch signalling controls pancreatic cell differentiation. Nature 400:877-881

46. Lee JC, Smith SB, Watada H et al (2001) Regulation of the pancreatic pro-endocrine gene neurogenin3. Diabetes 50:928936

47. Polak M, Bouchareb-Banaei L, Scharfmann R, Czernichow P (2000) Early pattern of differentiation in the human pancreas. Diabetes 49:225-232

48. Abbas-Terki T, Blanco-Bose W, Deglon N, Pralong W, Aebischer P (2002) Lentiviral-mediated RNA interference. Hum Gene Ther 13:2197-2201

49. Rubinson DA, Dillon CP, Kwiatkowski AV et al (2003) A lentivirus-based system to functionally silence genes in primary mammalian cells, stem cells and transgenic mice by RNA interference. Nat Genet 33:401-406

50. Stewart SA, Dykxhoorn DM, Palliser D et al (2003) Lentivirusdelivered stable gene silencing by RNAi in primary cells. RNA 9:493-501

51. Efrat S, Linde S, Kofod H et al (1988) $\beta$ Cell lines derived from transgenic mice expressing a hybrid insulin gene-oncogene. Proc Natl Acad Sci U S A 85:9037-9041 